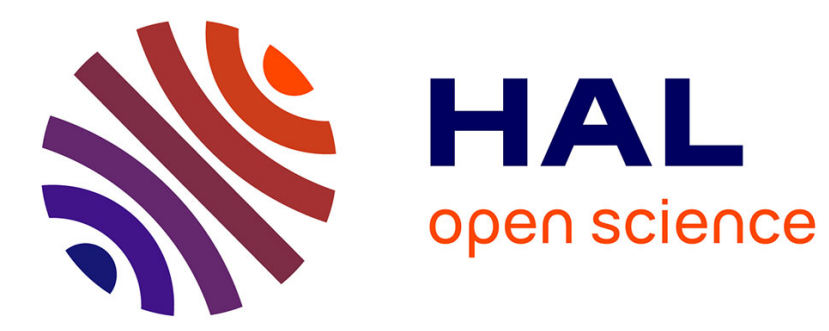

\title{
Quantitative microtomography: measurement of density distribution in glass wool and local evolution during a one-dimensional compressive load
}

\author{
Eric Badel, J-M Létang, G. Peix, D. Babot
}

\section{- To cite this version:}

Eric Badel, J-M Létang, G. Peix, D. Babot. Quantitative microtomography: measurement of density distribution in glass wool and local evolution during a one-dimensional compressive load. Measurement Science and Technology, 2003, 14 (4), pp.410-420. 10.1088/0957-0233/14/4/302 . hal-01609532

\section{HAL Id: hal-01609532 \\ https://hal.science/hal-01609532}

Submitted on 16 Mar 2018

HAL is a multi-disciplinary open access archive for the deposit and dissemination of scientific research documents, whether they are published or not. The documents may come from teaching and research institutions in France or abroad, or from public or private research centers.
L'archive ouverte pluridisciplinaire HAL, est destinée au dépôt et à la diffusion de documents scientifiques de niveau recherche, publiés ou non, émanant des établissements d'enseignement et de recherche français ou étrangers, des laboratoires publics ou privés. 


\title{
Quantitative microtomography: measurement of density distribution in glass wool and local evolution during a one-dimensional compressive load
}

\author{
E Badel ${ }^{1}$, J-M Létang ${ }^{2}$, G Peix ${ }^{2}$ and D Babot ${ }^{2}$ \\ ${ }^{1}$ LERMAB, ENGREF, 14 rue Girardet, 54042 Nancy Cedex, France \\ ${ }^{2}$ CNDRI Laboratory, INSA-Lyon, 69621 Villeurbanne Cedex, France
}

Received 15 November 2002, in final form and accepted for publication 28 January 2003

Published 4 March 2003

Online at stacks.iop.org/MST/14/410

\begin{abstract}
This paper proposes two applications of quantitative tomography. Assuming that the attenuation ratio of the $\mathrm{x}$-ray beam throughout the investigated sample can be evaluated with high accuracy, this paper deals with quantitative characterization of glass wool structure. In a first part, we measure the 3D spatial distribution of density in a sample that has been extracted from a glass wool plate. The calibration is performed and an experimental validation of the technique is realized. The heterogeneity of the material is characterized and explained in relation to manufacturing methods. In a second step, we follow the evolution of this distribution during a mechanical test. For this purpose, an original device has been developed in the laboratory in order to perform a tomographic process during a mechanical load. The proposed method compares the density distribution between two different compression steps and determines the evolution of density inside the sample. The local strain is evaluated along the stress direction. These first results allow assumptions about the relationships between structure and properties to be expressed.
\end{abstract}

Keywords: microtomography, x-ray, density, strain measurement, mechanical test, glass wool

(Some figures in this article are in colour only in the electronic version)

\section{Introduction}

X-ray tomography allows non-destructive evaluation of materials for a large set of purposes (Baruchel et al 2000, Grangeat 2000). Detection of voids, cracks and delaminations, geometrical arrangements of cells inside a foam, dimensional characterization of some hidden part, detection of precipitates, quantitative evaluation of a mixture and density measurements are thus feasible using transmission tomography based on measurements of x-ray attenuation throughout the evaluated object (Kak and Slaney 1988). A large set of scales is attainable, from the industrial scale (rocket propellers, $2.4 \mathrm{~m}$ in diameter), down to $0.7 \mathrm{~mm}$ samples scanned at the
ESRF (European Synchrotron Radiation Facility-Grenoble, France) with a $0.4 \mu \mathrm{m}$ voxel size. A great number of laboratory applications can be treated using standard cameras and x-ray tubes. The use of a microfocus allows design of a generalpurpose micro-scanner, able to perform tomographic imaging down to $5 \mu \mathrm{m}$ resolution typically, given the appropriate geometrical magnification and mechanical device.

It can be seen that among the enumerated fields of interest, a great number make use only of the dimensional observation of the images, involving qualitative information (Maire et al 2002). In the present paper, we propose to describe the evaluated object in a quantitative way, as performed on some advanced materials (Robert-Coutant et al 1999, 
Douarche et al 2001, Elliott et al 2002). We will explain first the physical basis and the specific characteristics of our xray scanner. The experimental process, including the gauging and the corresponding accuracy in the estimation of the local glass density, is described in section 3. Section 4 deals with mechanical tests. A tensile machine, consistent with the acquisition of the projection images, was designed to follow the local evolution of the density inside the sample during the compression test. Data analysis and interpretation of the mechanical behaviour based on the comparison of the glass wool volumes at two compression stages are also given in this part.

\section{Experimental set-up and acquisition parameters}

\subsection{General presentation of the experiment}

A 3D tomograph was designed and built, allowing an adjustable spatial resolution: the reconstructed $3 \mathrm{D}$ image of the object presents a cubic voxel, of which the size ranges from 8 to $100 \mu \mathrm{m}$. The tomograph is mainly constituted by a microfocus X-ray tube (Philips 161 HOMX) and an X-ray sensitive imager. The small size of the $\mathrm{x}$-ray tube focus allows us to proceed to a geometrical enlargement with a low value of the blurring (also denominated geometrical unsharpness). The focus size, which could be lowered down to $8 \mu \mathrm{m}$ on the HOMX-161 device, was simply set to $30 \mu \mathrm{m}$ for the present application, where the magnification is rather modest: 1.38. The imager, which consists in a low-noise CCD (charge-coupled device) camera cooled by a triple-stage Peltier cooler and equipped with a high-aperture lens, is used to catch the weak visible image emitted by a terbium-doped gadolinium oxysulfide $\left(\mathrm{Gd}_{2} \mathrm{O}_{2} \mathrm{~S}[\mathrm{~Tb}]\right)$ fluorescent screen. For the present application, the active size of the fluorescent screen is $100 \mathrm{~mm} \times 100 \mathrm{~mm}$. Displaying the whole scene on the $1024 \times 1024$ pixels of the CCD chip, every pixel is equivalent to nearly $100 \mu \mathrm{m}$, at the level of the screen. The scheme of the constructed device is displayed in figure 1 .

\subsection{Choice of the different instruments}

The HOMX-161 X-ray tube, constructed by the Philips Company, is a microfocus $\mathrm{X}$-ray tube which allows a dissipation of $8 \mathrm{~W}$ on the tungsten anode in the fine-focus configuration $(8 \mu \mathrm{m})$. The high voltage, of which the value is adjustable between 20 and $160 \mathrm{kV}$, is regulated with a very high accuracy: the residual ripple (relative fluctuation of the voltage) is lower than $2 \times 10^{-4}$. The main interest of a microfocus $x$-ray tube lies in the very low geometrical unsharpness generated in the image. A direct enlargement of the image, achieved by bringing the sample close to the focus (and away from the camera), is thus allowed. In such a case, the stability of the $\mathrm{x}$-ray tube becomes a major limitation, as any change in the position of the focus, during the dataset acquisition, leads to misalignment, with the axis of rotation of the sample and with the camera. The use of a fine metallic ball, set to the scanner's structure, would allow us to keep track of the drift and thus to correct it by software. Such an opportunity is not used for the present application where the slight displacements of the focus (a few microns) are far lower than the selected focus size $(30 \mu \mathrm{m})$. In the case of the present application, a very low
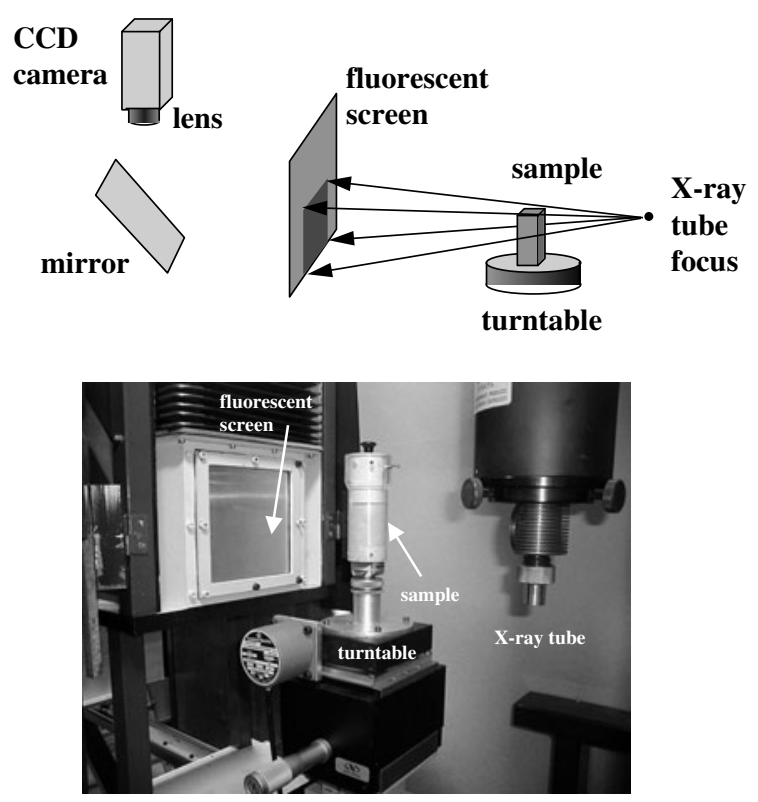

Figure 1. General scheme and photograph of the x-ray scanner; the sample on the turntable is set inside the compression device.

tube voltage was selected $(24 \mathrm{kV})$, as the imaged specimen, of low density, is characterized by a low x-ray attenuation.

The active part of the $\mathrm{x}$-ray sensitive imager is, as shown in figure 1, the fluorescent screen. The choice of the fluorescent material was determined by the high density, the high atomic number and the high conversion yield of $\mathrm{Gd}_{2} \mathrm{O}_{2} \mathrm{~S}$. Thus, a very thin layer of fluorescent material ( $80 \mu \mathrm{m}$ in our case) is sufficient to deliver an acceptable image of the object. Increasing the thickness of the layer beyond this value will of course enhance the light emission but will blur the image, due to diffusion of visible light inside the screen and to selfabsorption of the emitted light. A rule of thumb in the choice of the screen thickness is that the lateral resolution is roughly equal to 1.2 times the thickness of the screen, with $\mathrm{Gd}_{2} \mathrm{O}_{2} \mathrm{~S}$. The screen thickness selected for our application is thus consistent with the $100 \mu \mathrm{m}$ pixel size of the camera. Another predominant parameter in the choice of the fluorescent compound is the wavelength of the emitted visible light: $550 \mathrm{~nm}$ in the case of $\mathrm{Gd}_{2} \mathrm{O}_{2} \mathrm{~S}$, which is fairly well adapted to the domain of sensitivity of the CCD chip.

This image is caught by the CCD camera and its associated lens; the mirror allows us to set the CCD apart from the main $\mathrm{x}$-ray flux. As the solid angle under which the lens is seen from every point of the screen is low, a high efficiency of the CCD and a high aperture of the lens are needed. The former point is taken into account using a thinned back-illuminated chip (sensitivity $80 \%$ instead of $35 \%$ for a conventional chip). The latter leads to the use of a high-quality lens, as geometrical distortions and spherical aberration must remain acceptable in spite of the high aperture. An AF-Nikkor lens, $85 \mathrm{~mm}$, $f / 1.4$ was chosen. Selecting the 2.8 aperture only, we are able to keep the blurring to an acceptable level, in the peripheral part of the image, and to provide a sufficient depth of focus, as regards the potential unflatness of the relatively large $(100 \mathrm{~mm} \times 100 \mathrm{~mm})$ screen. Using a $14 \mathrm{~mm}$ extension ring allows us to focus perfectly on the fluorescent screen, settling the distance to the value ' $1.3 \mathrm{~m}$ ' on the adjustment ring 
of the lens, while the real distance between lens and screen is around $420 \mathrm{~mm}$. Such care is essential for the preservation of a low value of image aberration because lenses are fitted to 'long-distance' rather than 'short-distance' focusing. In our case, aberration and distortion of the image remains tolerable and do not need any software correction. Besides, the need for a high dynamic range imposes a reduction of all electronic noises inside the camera, like the readout noise and the noise associated with the dark current. The former is kept small using a slow-scan camera and frame grabber. The later is reduced using a cooled CCD chip. A triple-stage Peltier cooler and a cooled-water circulator allow us to reach the temperature of $225 \mathrm{~K}$ at the level of the chip. The selected camera is the Hamamatsu C-4880, equipped with a 'SITE 003 A' chip $(1024 \times 1024$ pixels; pixel size $24 \mu \mathrm{m} \times 24 \mu \mathrm{m})$ and a sealed vacuum. The main characteristics of our equipment have been described elsewhere (Cendre et al 1999).

The device is completed by a motorized turn-table that allows us to rotate the sample: $N$ images, named projections, are captured with an angular increment of $360 / N^{\circ}$. Image reconstruction is effected using the three-dimensional FBP (filtered backprojection) algorithm of Feldkamp (Feldkamp et al 1984).

\subsection{Experimental setting for glass wool imaging}

The samples, with a square section, were cut in an industrial glass wool plate. On each sample, 900 projections were acquired on a whole turn (pitch: $0.4^{\circ}$ ). Table 1 summarizes the experimental parameters selected for this application.

It must be noticed that the magnification was rather low ( $G=1.381)$ and that the voxels were binned by packs of four in the three directions (super-voxels containing 64 elementary voxels). The reason is that the modelling of the glass wool and the track of density evolution do not require an accurate resolution. A spatial resolution able to track the individual glass fibres would be inefficient because the total reconstructed volume would be too small and not representative of the mechanical compressive process as a whole. The size of the generated voxel is $280 \times 280 \times 280 \mu \mathrm{m}^{3}$. This volume is big enough to detect a great number of photons in a few seconds, but it stays small in comparison with the size of the heterogeneity of the structure.

Applying those conditions, the acquisition of a 'white' image (i.e. realized without any sample) generates 12500 electrons in the well of every CCD pixel, the noise being equal to $14 \mathrm{e}^{-}$rms. Binning the pixels, as mentioned above, improves the dynamic range of the camera.

\section{Local density measurement}

The principle of tomography has been presented in a great number of books and papers and some have already been referenced in the introduction. The basic principle is that the transmitted intensities, detected using an imaging device with a linear response, are related to the integral attenuation of the various materials encountered inside the object, along each individual x-ray beam. For a specific photon energy and for every sensitive pixel in the detector, the intensity is given by the Beer-Lambert law:

$$
I=I_{0} \exp \left(-\int_{\text {ray path }} \mu(x) \mathrm{d} x\right)
$$

where $\mu(x)$ stands for the attenuation coefficient of the inspected material at that energy along direction $x$ of the ray path. Measuring $I$ and $I_{0}$ with the imaging device along a great number of paths, we are then able to determine the corresponding integrals of $\mu(x)$. It was demonstrated in 1917 by Radon that, using this set of integrals, the $\mu$ map of the object can be reconstructed. That point is the mathematical basis of tomographic reconstruction. It must be noticed that recent syntheses were published about 3D reconstruction methods (Grangeat 2001).

It is of main importance to determine the physical meaning of $\mu$. The interest of attenuation measurements lies in the fact that, for a given compound (mainly $\mathrm{SiO}_{2}$ in our case), $\mu$ is dependent on photon energy and proportional to $\rho$, the density of the studied material. It thus appears that, for a given material and a given energy, $\mu / \rho$ is a constant. A problem, in practice, arises from the fact that the $\mathrm{x}$-ray beam delivered by an x-ray tube is strongly polychromatic. Throughout the path inside the sample, low-energy components of the spectrum are therefore attenuated preferentially, leading to a relative increase of the 'hard' components of the spectrum. Such behaviour, named beam hardening, causes different kinds of artefact in the reconstructed map. The most cumbersome is the cupping effect whose influence can be eliminated using a software correction based on a calibration effected on a step wedge of the same material (Douarche et al 2001).

\subsection{Calibration in density}

The selected calibration method is purely experimental; it consists in imaging a step wedge, machined in a material with a constant density and presenting the same chemical composition as the glass wool sample. Unfortunately, the glass wool is in fact composed of glass fibres and of organic binder. Assuming that the binder is present in small quantities (a few per cent) and produces a low effect on attenuation properties (due to its low atomic number), we used, for the step-wedge, homogeneous glass plates, whose composition is close to that of fibres. Six thicknesses were available: $0.13,0.17,0.51$, $1.03,2.09$ and $3.12 \mathrm{~mm}$. Simple projection images were acquired and attenuation ratios were evaluated (cf figure 2).

This allows the equivalence between thickness and $I / I_{0}$ ratio to be determined (figure 3 ). The experimental data are well fitted by a polynomial approximation. Rewriting the classical attenuation law and taking into account the fact that $\mu / \rho$ is a constant, we get

$$
\ln \left(\frac{I_{0}}{I}\right)=\frac{\mu}{\rho} \int \rho(x) \mathrm{d} x .
$$

In the case of gauging using the step-wedge, $\rho$ is constant, and equation (2) shows that the $I / I_{0}$ ratio is correlated to the integral of $\mathrm{d} x$ all along the path (i.e. to sample thickness), whereas in the glass wool sample, every transmission measurement leads to the determination of the integral of 

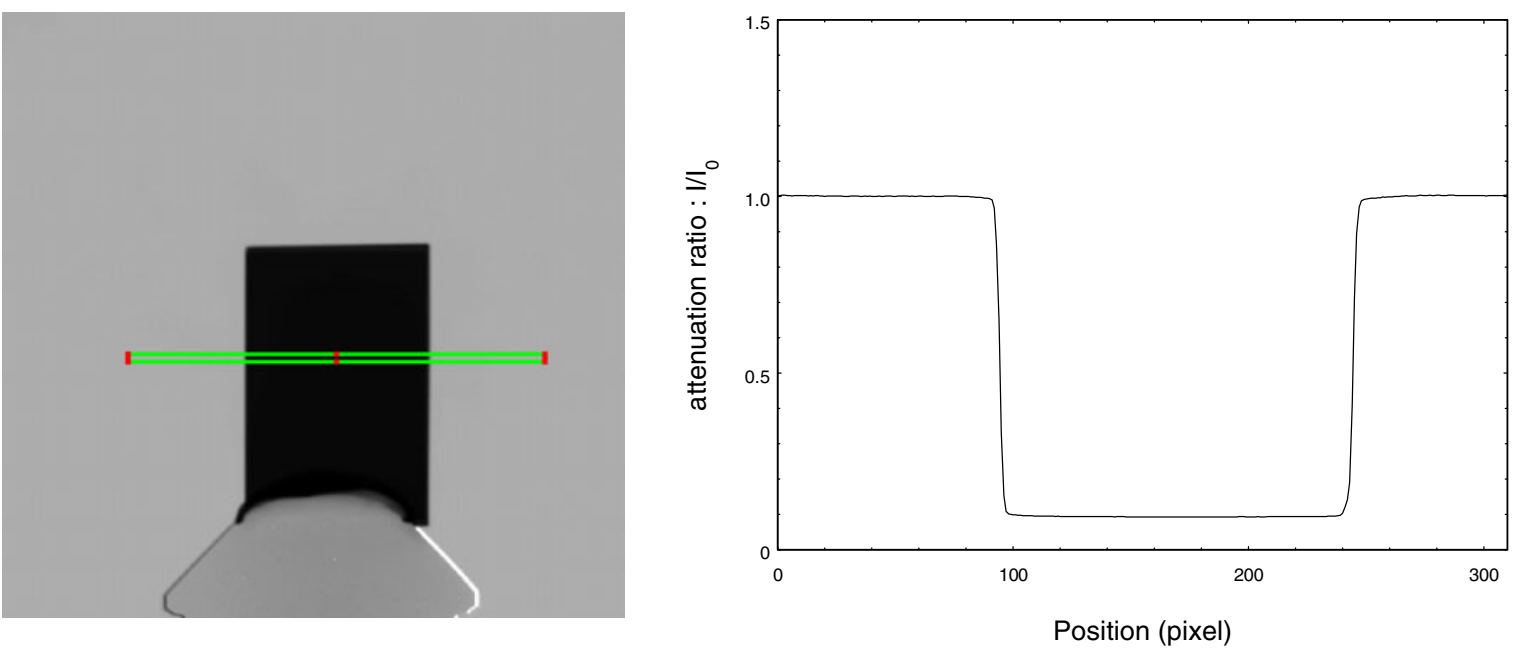

Figure 2. Image of a glass sample (on its support) and attenuation profile used for the $I / I_{0}$ ratio measurement.

Table 1. X-ray imaging experimental conditions.

\begin{tabular}{|c|c|c|c|c|c|c|c|c|c|c|}
\hline \multicolumn{2}{|c|}{$\begin{array}{c}\text { Samples } \\
\text { dimensions }\end{array}$} & \multicolumn{2}{|c|}{$\begin{array}{c}\text { X-ray tube: } \\
\text { energy, intensity }\end{array}$} & \multicolumn{3}{|c|}{ Exposure time } & \multicolumn{4}{|c|}{ Volume reconstruction } \\
\hline $\begin{array}{l}\text { Size } \\
(\mathrm{mm})\end{array}$ & $\begin{array}{l}\text { Height } \\
(\mathrm{mm})\end{array}$ & $\begin{array}{l}\mathrm{HV} \\
(\mathrm{kV})\end{array}$ & $\begin{array}{l}\text { Current } \\
(\mathrm{mA})\end{array}$ & $\begin{array}{l}\text { Exp. } \\
\text { (s) }\end{array}$ & $\begin{array}{l}\text { Exp. } \\
+ \text { readout (s) }\end{array}$ & $\begin{array}{l}\text { Total time } \\
\text { for } 900 \text { proj }\end{array}$ & $\begin{array}{l}\text { Magnification } \\
G\end{array}$ & $\begin{array}{l}\text { Voxel size } \\
(\mu \mathrm{m})\end{array}$ & $\begin{array}{l}\text { Voxel size } \\
\text { after binning } \\
(\mu \mathrm{m})\end{array}$ & $\begin{array}{l}\text { Reconstructed } \\
\text { volume (pixels) }\end{array}$ \\
\hline 40 & 60 & 24 & 0.3 & 5.5 & 10.5 & $\begin{aligned} & 9450 \mathrm{~s} \\
\approx & 2 \mathrm{~h} 40 \mathrm{~min}\end{aligned}$ & 1.381 & 70.2 & 280.9 & $165 \times 165 \times 227$ \\
\hline
\end{tabular}

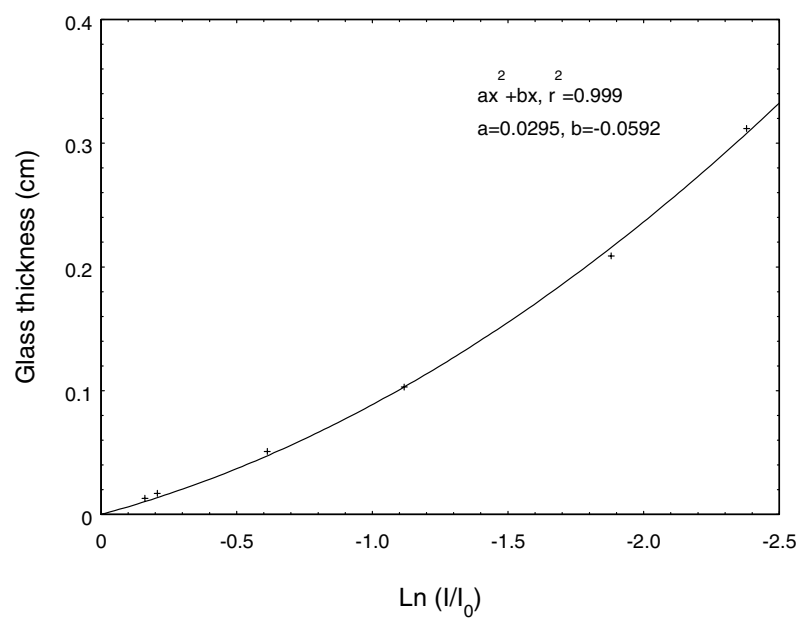

Figure 3. Calibration measurements: evolution of the attenuation ratio $\left(I / I_{0}\right)$ according to the thickness of glass.

density. The reconstruction process, which consists in the resolution of the inverse problem, thus delivers a 3D map of density. Figure 4 represents the typical structure of glass wool product. These views are extracted in the three characteristic planes of the material. The $X$ direction corresponds to the advancing direction of the conveyor belt involved in the manufacturing process. The $Z$ direction is perpendicular to the glass wool plate. The general anisotropy of fibre orientation is clearly displayed.

The correction of beam hardening is also possible using more advanced methods. For instance, attenuation of the
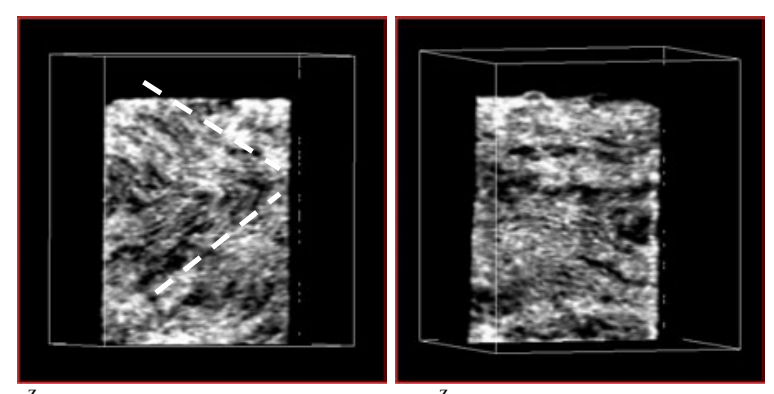
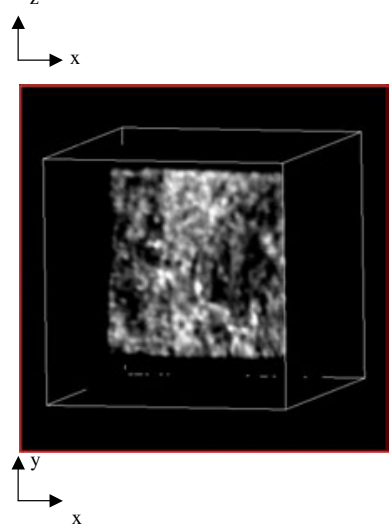
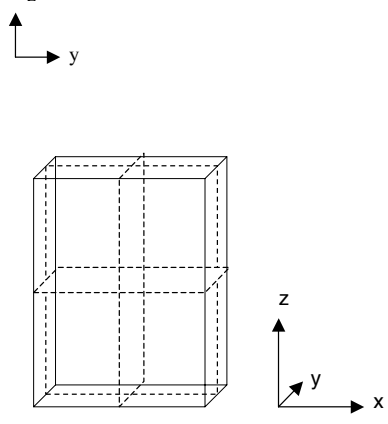

Figure 4. Density distribution in the three main planes of the glass wool plate; $X$ corresponds to the conveyor belt direction and the $Z$ direction is perpendicular to the plate.

different spectrum components can be calculated, provided the tube spectrum is known (de Paiva et al 1998). In our case, as 


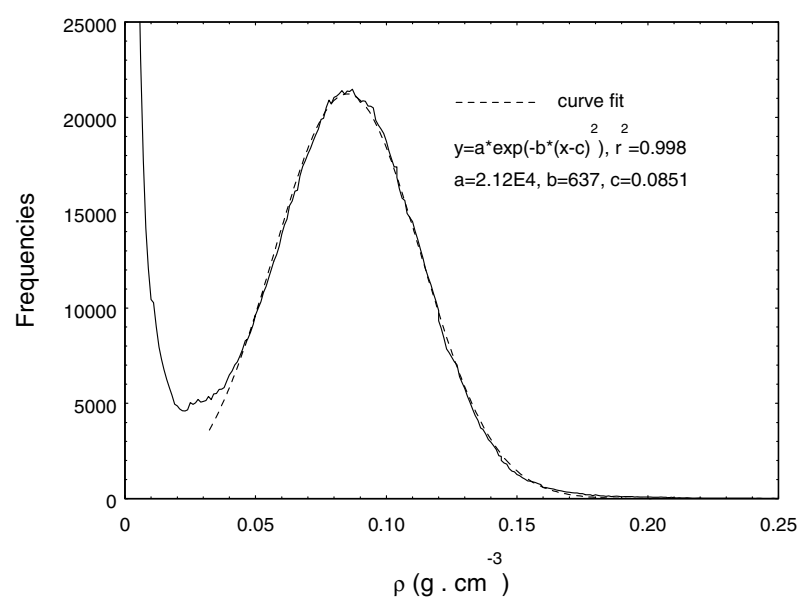

Figure 5. Density histogram. $N$, the number of voxels per density unit, is displayed as a function of density. The peak around the zero value corresponds to air surrounding the sample.

the selected tube voltage is rather low $(24 \mathrm{kV})$, we did not find any available data, in the literature, about the tube spectrum. Its experimental determination being not straightforward, we preferred to rely on an experimental calibration.

\subsection{Qualitative observation and quantitative evaluation}

Quantitative measurement requires noise evaluation. Several effects can damage the measurement: during the recording of the images (photon statistics) or during the reconstruction process (artefacts). Experimental evaluation of total noise is carried out. A volume outside the glass wool sample is isolated. In this volume of air, the density histogram is calculated and fitted with a Gaussian model: the standard deviation is $6.74 \times 10^{-4} \mathrm{~g} \mathrm{~cm}^{-3}$. Taking into account a Poisson distribution model for the $\mathrm{x}$-rays impinging the detector, we can estimate the corresponding noise inside the wool, which gives $1.8 \times 10^{-3} \mathrm{~g} \mathrm{~cm}^{-3}$.

The histogram of the whole reconstructed volume (figure 5) presents a high peak near the origin, which corresponds to air. The second peak, whose average value is evaluated to $0.085 \mathrm{~g} \mathrm{~cm}^{-3}$, is the glass wool contribution. The Gaussian model, which fits this peak very well, delivers a $0.028 \mathrm{~g} \mathrm{~cm}^{-3}$ standard deviation value, which quantifies the density heterogeneity since the photonic noise $(1.8 \times$ $10^{-3} \mathrm{~g} \mathrm{~cm}^{-3}$ as mentioned above) is much smaller.

Observations of slices extracted from the three main planes give information about the structure. $X Z$ and $Y Z$ planes show structures with stratum of high or low density. They stand horizontal or locally inclined. The $X Z$ plane shows a ' $\mathrm{V}$ ' structure that results from a shear in the $X$ direction during the manufacturing process. This stress occurs during the first hot compression of the material, just after the mix (fibre + binder) is deposited. It permits a better entanglement of fibres and a better cohesion between the different levels inside the thickness of glass wool sheet. The structure of the $X Y$ planes, in parallel lines, results from this manufacturing step too.

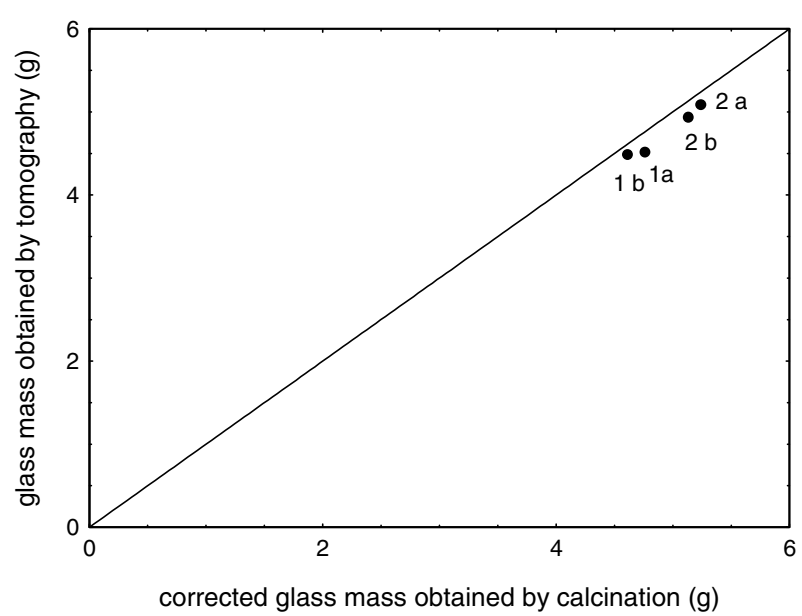

Figure 6. Comparison of the estimated mass, using x-rays, with the mass of the calcined sample (glass only).

\subsection{Experimental validation of density measurements by $x$-ray}

The validation procedure is done by comparison with weight measurements. Four samples are used for a 'blind test' (two couples extracted from two different industrial plates). On the one hand, the complete tomographic process (including the calibration step) is performed for each sample. Once the local density is recovered for each voxel, we are able to compute the total mass of the sample using a numerical summation. On the other hand, the samples are weighed using a precision balance (accuracy: $0.1 \mathrm{mg}$ ). The results show that, using x-ray tomography, the mass of the four samples was systematically under-evaluated with a 9-10\% error. The relative order of the four masses was preserved. In order to confirm these results, we performed the same experiments a second time: the same behaviour was observed. Moreover, these new experiments confirmed that the density evaluation using x-ray tomography could be a very reproducible process.

In fact, binder causes the major part of this systematic deviation. Binder, which represents around $6 \%$ of the total weight, is in practice not detected by x-rays. In the low-energy range, $x$-ray attenuation is proportional to $Z^{3}, Z$ being the atomic weight of the evaluated material. The difference in $Z$ between glass and binder is quite important, since glass wool is mainly constituted by $\mathrm{SiO}_{2}$ (with $\mathrm{Si}, Z=14$ and $\mathrm{O}, Z=8$ ) while the binder is modelled by the formula $\mathrm{C}_{x} \mathrm{H}_{y} \mathrm{~N}_{z}$ (with $\mathrm{C}, Z=6 ; \mathrm{N}, Z=7$, and $\mathrm{H}, Z=1$ ). Glass fibres therefore mainly attenuate $\mathrm{x}$-rays: a quick computation shows that $99 \%$ of the attenuation inside the sample is due to glass fibres.

A new estimation was therefore established: calcining the sample at $450^{\circ} \mathrm{C}$ for $2 \mathrm{~h}$ before weighting allows the precise determination of glass mass alone. The comparison with mass estimated using X-rays is therefore better (figure 6). However, it must be considered that those two quantities cannot be strictly compared, since the binder contributes slightly to the total attenuation $(\approx 1 \%)$. The corresponding correction would consist in decreasing the estimated mass by $1 \%$ in figure 6 : therefore, tomographic measurement still underestimates the glass mass obtained after calcination by a factor in the range $2.5-5 \%$. 

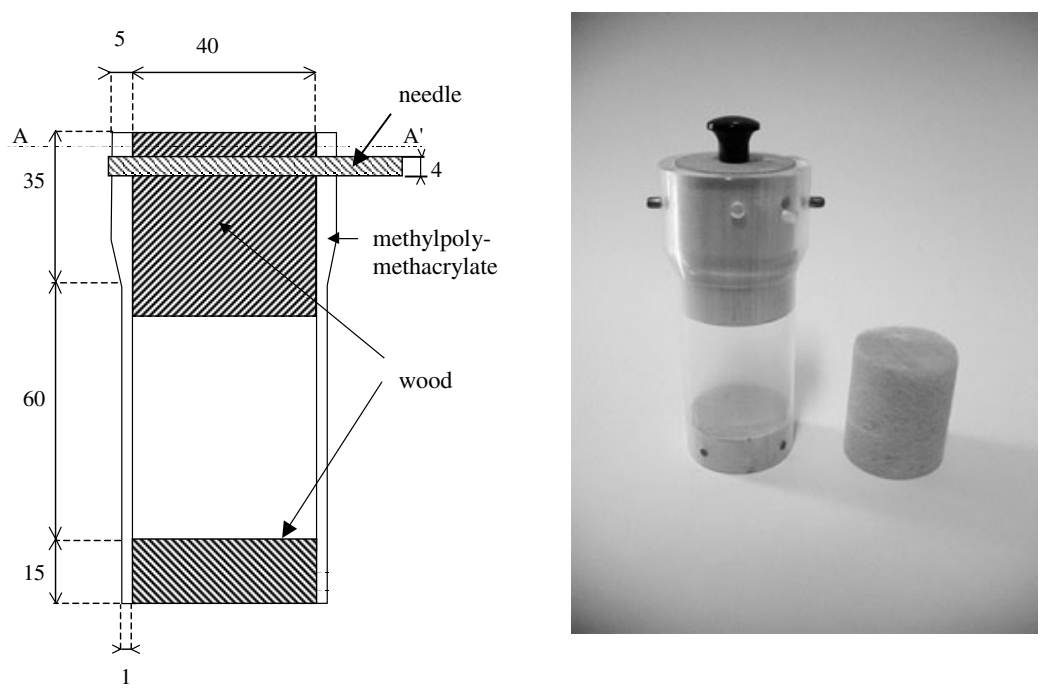

Figure 7. Compression device: (a) general scheme; the bottom extremity is fixed to the tube and the top extremity slides vertically between two pre-selected positions, (b) photograph showing the device and the sample.

On the four examples displayed in figure 6, this last error appears to be systematic. It must however be noticed that a difference exists between both types of glass constituting respectively the fibres and the plates: elaboration of both materials was performed using different temperature cycles. A slight difference of concentration of the high- $Z$ impurities which are necessarily present inside glass explains a noticeable error in the estimation of mass using x-rays, due to the term $Z^{3}$ already quoted.

\section{Mechanical test}

In this part, we intend to follow the evolution of glass distribution during a compressive load. In this study, the case of a 1D stress perpendicular to the plate is described. This is the natural way to load this industrial product. Initially, great compression under hot conditions occurs during the manufacturing process and explains the characteristic structure we observed previously. Then, several compressive loads, still perpendicular to the plate, commonly happen during the life of the product (storage, laying, ...). The strain can easily reach high values in the range $20-25 \%$. The followup of density variation by x-ray methods leads to several problems. On the one hand, all the physical points of the material are supposed to move. On the other hand, in such a heterogeneous material, local density could vary considerably according to the local properties and the local stress. These modifications are detected by x-ray tomography. It becomes almost impossible to locate and to follow one physical point. This is a fundamental difference with the processes that are commonly used to determine local strain fields using images coming from optic or electronic microscopy (Badel 1999, Farruggia and Perré 2000, Tatschl et al 2000). For these techniques, the grey value of one pixel is usually not supposed to change drastically. Within the frame of the present study, we developed a method that makes it possible to get free of the determination of the local density change and of the complete local strain field computation.

\subsection{Requirements and design of the experimental load machine}

The design of the experimental device has to follow several mechanical requirements.

- The stiffness of the device has to be high enough in order to withstand a load of few kilograms without significant deformation.

- The shape of the device has to ensure that the strain field is close to a one-dimensional deformation.

- It has to be small and light in order to be installed on the small turntable of the microtomograph.

On the other hand, several additional requirements are added by considerations about x-ray detection.

- Attenuation of x-rays by the device itself must remain tolerable: it must be kept in mind that we use low-energy photons, easily attenuated inside matter.

- The nature of the material and the shape should not perturb the reconstruction process, thus generating artefacts.

- The design must nevertheless allow the detection of the device during the analysis step.

According to these requirements, the compressive machine is mainly made of methyl-polymethacrylate. It is a stiff and homogeneous material. Furthermore, it permits the sample to be visualized by the user during the experiment. The device is composed of two parts. The shape is a tube, $110 \mathrm{~mm}$ in height, which makes it possible for the sample to be guided during the compression step. The internal diameter is $40 \mathrm{~mm}$. In the bottom part, where the sample is set, the wall is very thin $(1 \mathrm{~mm})$ in order to minimize the $\mathrm{x}$-ray attenuation, whereas in the top part, which is outside the detection cone, it is thicker in order to withstand the mechanical load. A precise dual-calibration study (methyl-polymethacrylate and glass) revealed that the compression device can be neglected in the glass density calibration since the overestimation in the density value is within a few per cent. 


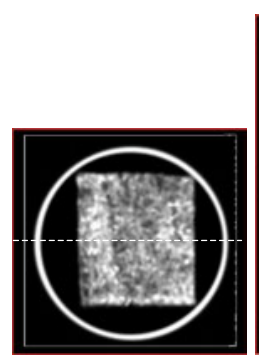

a

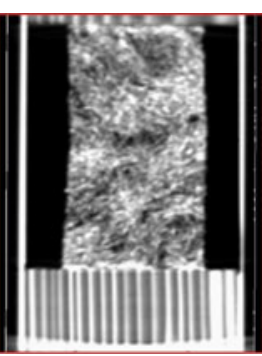

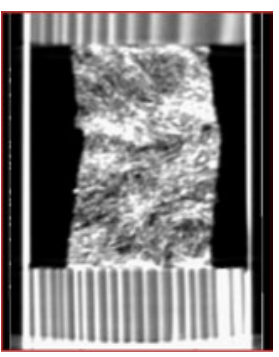

Figure 8. (a) Position of the sample (case of a sample with a square section) in the compression device; (b) cross section before compression; (c) cross section after compression. The buckling of the structure is visible on the last image.

A piece of wood, $15 \mathrm{~mm}$ thick, closes the tube at the bottom extremity. This choice of material is motivated by the very good compromise between density and rigidity. On the one hand, the low density that is 'close' to the glass wool does not generate reconstruction artefact. On the other hand, the Young modulus $(\approx 10000 \mathrm{MPa})$ is high enough to ensure a very stable position of the stand. An another piece of wood, $50 \mathrm{~mm}$ thick, slides at the top part of the tube in order to force the displacement of the glass wool sample (figure 7). The load is performed manually by the user, with a perfectly vertical motion. A needle goes right through the diameter and ensures a precise location of the cap, and thus a precise settlement of a constant strain level.

Two positions have been pre-selected. The first one corresponds to the initial state. A low load is set to ensure the contact and to position the sample. The second one corresponds to the compression state. The macroscopic strain is about $8 \%$.

\subsection{Design of the sample and global density evolution}

Industrial glass wool plates are several metres long and wide and only a few centimetres thick. During their transversal compressions (manufacturing or storage steps), the displacement occurs mainly in this transversal direction, as in semi-infinite materials. In order to simulate these special conditions for a small sample, the compressive process has to ensure that load and displacements stay in the right direction. Several tests proved that buckling occurs easily when the sample is not bounded. Figure 8 shows the case of a sample with a square section. Despite the care of the user, the great initial heterogeneity produces a local collapse that spreads the sample and the structure buckles. This lateral transfer of matter does not correspond to a desired 1D strain field.

The solution we opted for consists in a cylindrical sample that fits the tube size (figure 9). The tube guides the macroscopic displacement. A small space $(<1 \mathrm{~mm})$ between sample and methyl-polymethacrylate surface minimizes the friction and makes it possible for a potential Poisson behaviour to occur.

The two histograms, before and after compression (figure 10), make it possible to visualize the evolution of density distribution. The average density is calculated before $\left(81.316 \mathrm{~kg} \mathrm{~m}^{-3}\right)$ and during compression $\left(87.968 \mathrm{~kg} \mathrm{~m}^{-3}\right)$. These values correspond to an average compression strain

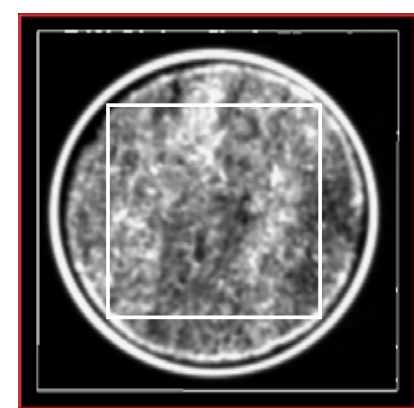

Figure 9. Design of the cylindrical sample and position in the compression device. The square illustrates the measurement area.

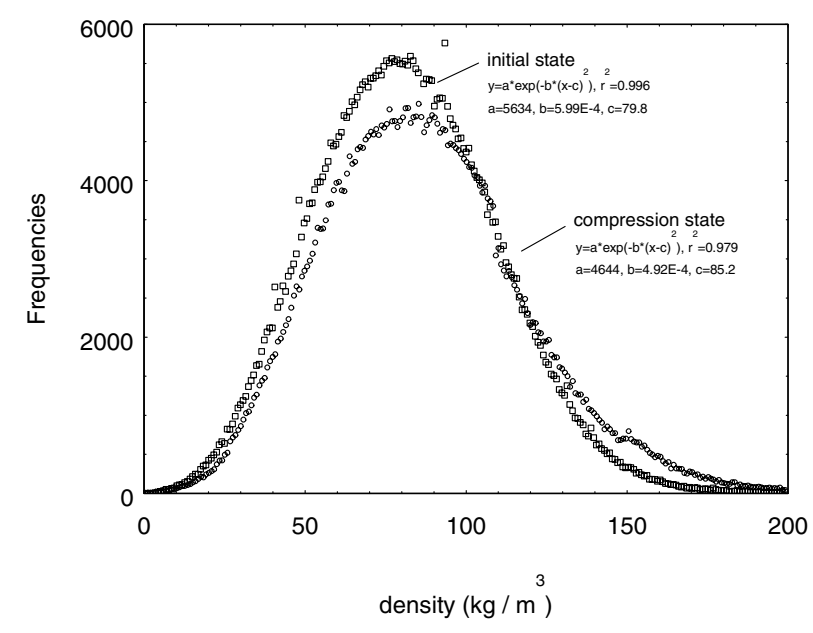

Figure 10. Evolution of density distribution during compression.

equal to $7.6 \%$, which is close to the $8 \%$ desired value. Unlike in the case of typical multilayer composites, where deformation occurs mainly in the soft parts during a tensile or compressive test, we rather observe a uniform translation of the frequency histogram towards the high-density values. The entire sample seems to be affected even in those areas where glass density is already high.

\section{3. 'Local' densification analysis process}

The determination of the densification cartography leads to the resolution of the complete strain field in the sample. Two kinds of hypothesis are commonly used in image analysis. The first one assumes that the grey level of a physical point of the sample does not change during the experiment (Badel 1999, Farruggia and Perré 2000). This makes it possible for all points to be located and their displacement to be measured. The other assumes that the strain is uniform in a small volume. A few particular points are detected and correlation processes are performed in their neighbourhood.

We opted for a simple and robust method that frees the detection of specific points. The principle consists in evaluating the mass of a volume and following it during the compression step. The principle is based on a 1D analysis, which is in accordance with the design of the experimental device. After the reconstruction operation, the volume is easily split into slices one voxel in thickness along the compression direction. The mass of each one is then computed. The same process is performed for the two states: before and during 


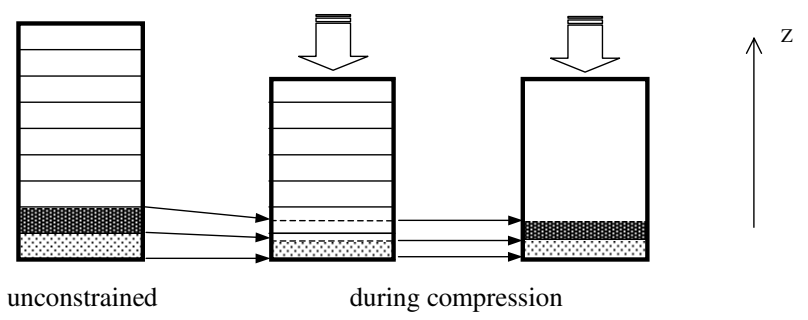

Figure 11. Computation of strain by mass conservation. The new thickness of a unitary slice is evaluated in order to keep the same matter quantity during the compression.

compression. Assuming that the mass of the first slice is well known in the unconstrained sample, the corresponding volume is determined in order to ensure the mass conservation during the compressed state. This defines a new thickness that allows the local compression strain to be evaluated (figure 11). The result is interpreted as the average deformation for the individual slice.

This complete process is reiterated for the entire sample along the compression direction and permits us to determine the compression strain for each slice. The robustness of this algorithm is due to the fact that it is totally free from point detection and is based on direct measurements of matter quantity. The control of potential drift is intrinsic to the method: at this end of the process, the total masses before and during compression should be equal and the entire volumes should be scanned. The essential condition that makes this process possible is the reproducibility of the measurement. This is ensured thanks to the high signal to noise ratio that is reached using the binning operating conditions (see section 2.3).

This process is not performed on the entire sample. Because of the cylindrical shape and the very thin space between glass wool and the wall of the tube, only the central square part is taken into account (figure 9). The implicit assumption that follows is that no great lateral mass transfer occurs from this central part towards external part during the compression test.

\subsection{Measurement of density distribution variation}

Integration of each mass distribution profiles (figure 12) allows control of the mass conservation. The slight difference between the two measurements, $0.7 \%$ of the total mass of the sample, is lower than the mass of one individual slice and can be attributed to the description of the volume using a discrete number of voxels.

The mass distribution curves (figure 12) show that initial density is significantly higher at the top and at the bottom of the sample and lower in the core region. These profiles are observed on other composites such as wood fibreboard (Papadopoulos and Traboulay 2002, Wang et al 2001) and are commonly involved in the hot pressing process during manufacture. A first comparison between the two states indicates that this characteristic seems to be exacerbated during the compression.

The computation of compression strain starts at the bottom of the sample and runs towards the top along the $Z$ direction. Figure 13 illustrates the results of the densification analysis. The mean value of the compression strain is $8.7 \%$ and is in agreement with the macroscopic displacement that is fixed at the top face. The compression strain curve confirms that density is reinforced near the board faces. In these regions, the strain exceeds $12 \%$.

The similitude of the compression strain curve with the density profiles is patent. The shape is globally analogous. It suggests a direct relationship between density and behaviour. However, simple analysis shows that this simplistic 'densitycompression strain' correlation is not available. A lag is perceptible and shows that compression could increase when density is decreasing. This is particularly patent at the top of the sample and in a small area of the core region (figure 14). This leads us to consider the local structure of the material as an explaining parameter. For instance, a fine observation of the sample shows that in this particular region of the glass wool sample, the material looks locally like a sequence of light/dense/light/... areas. In this case, we notice a classic behaviour of a multi-layer composite and deformation occurs preferentially in low-density areas.

In contrast, it could be surprising to observe very low deformation in the low-density area. In principle, this contradicts the previous analysis. At this step, only visual inspections on the lateral sides lead us to remark the presence of binder that occurs in the empty space and locally reinforces the structure. This great concentration of binder is marked by a very specific yellow colour that is visually observed. A definitive confirmation of this analysis would require the complete determination of binder distribution inside the glass wool sample.

Finally, the computation of the compression strain is applied along the two lateral directions. Figures 15 and 16 permit us to show that the profiles are identical and that no significant deformation occurs. It confirms that no mass transfer is observable in these directions. The consequence of the vertical compression is definitely a collapse of the porous structure along this same direction.

\section{Conclusion and prospects}

$\mathrm{X}$-ray tomographic tools have been used in a quantitative way. Thanks to a compromise with spatial resolution, signal to noise ratio has been increased enough to detect very low density variations with a good accuracy. Pre-calibration allowed glass wool density distribution to be evaluated, and heterogeneous structure of the glass wool plate to be observed. However, the presence of a second component, the binder, generates a slight under-estimation. A better description would require dual-energy experiments in order to dissociate the detection and to consider its effect.

The high reproducibility of the measurements allows the comparison between two mechanical states. The compression device we built reproduces a 1D strain condition. Comparison and analyses of the data are based on matter conservation. It produces a stable process that is well adapted to $\mathrm{x}$-ray detection. The results show very heterogeneous behaviours. Just as during the manufacturing process, the density is reinforced near the top and bottom faces. In the other parts, the initial local density alone cannot explain the local behaviour. The macroscopic and local structure of the material, which results from the manufacturing process, has to be 


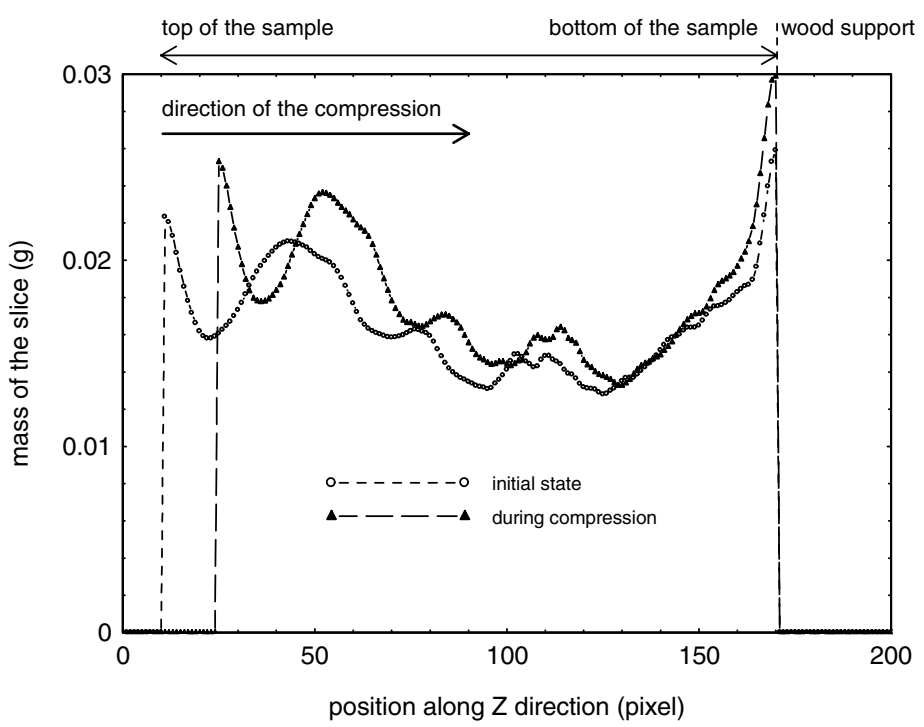

Figure 12. Mass profiles of the unitary slices (thickness $=1$ voxel). Comparison between initial and compression states.

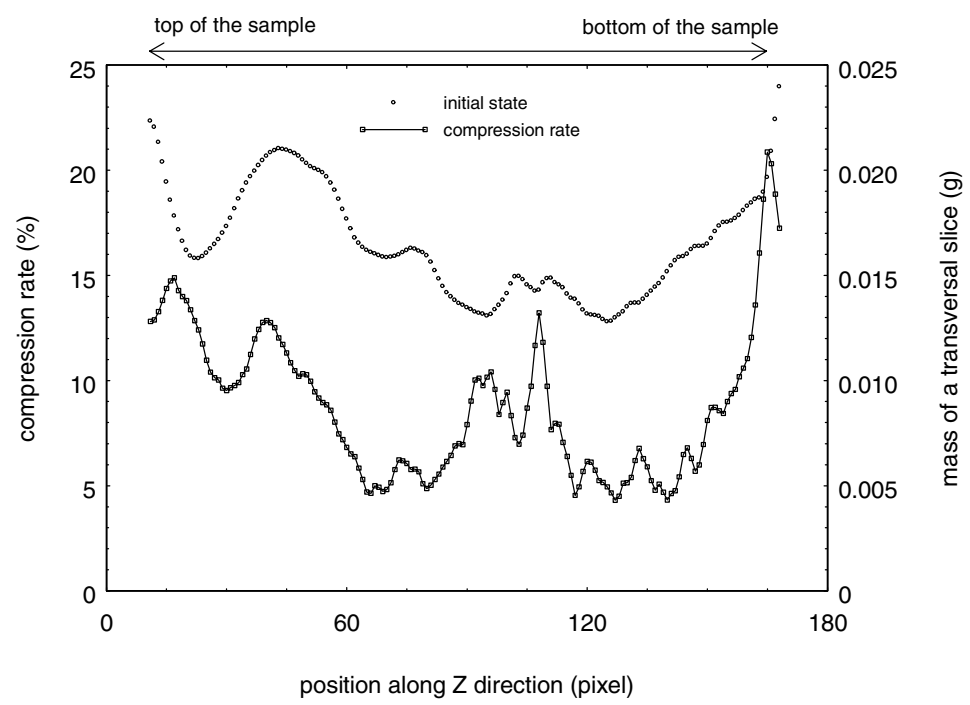

Figure 13. Compression strain profile along the $Z$ direction.

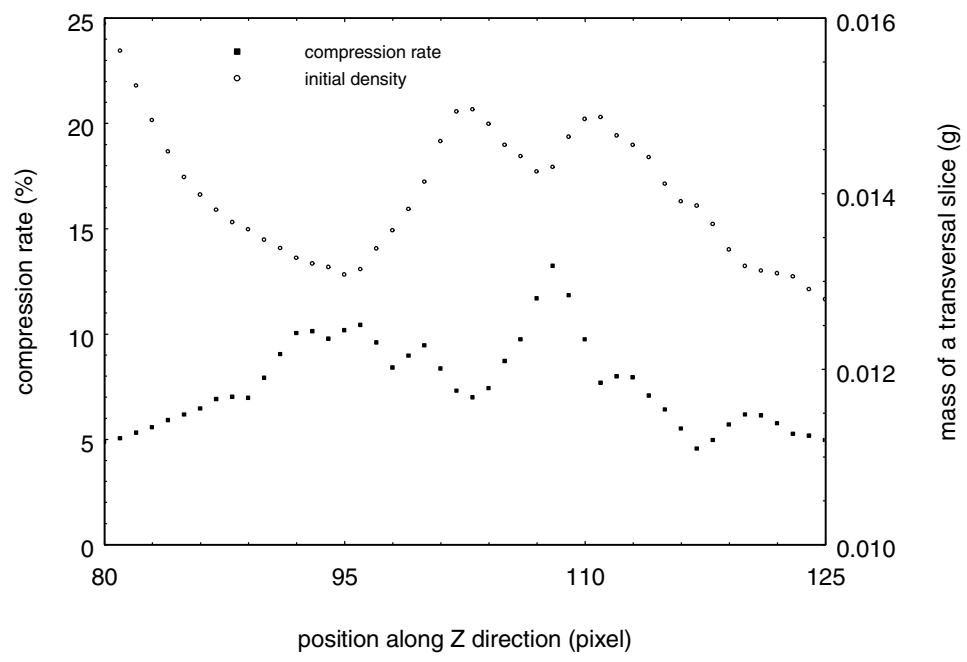

Figure 14. Initial density profile and compression strain in the core region (profiles along the $Z$ direction). 


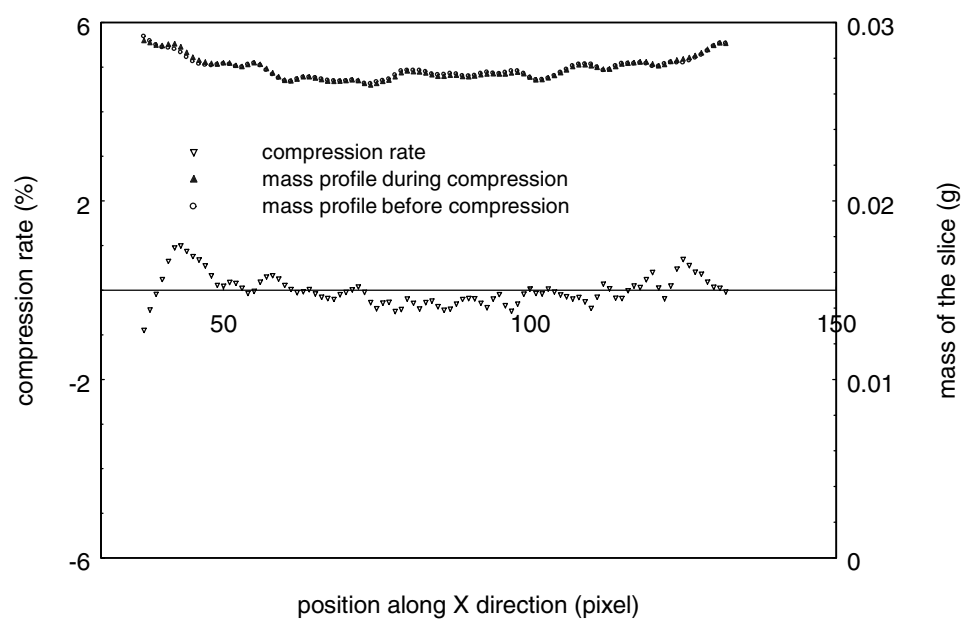

Figure 15. Density (before and during compression) and compression strain profiles (along $X$ direction).

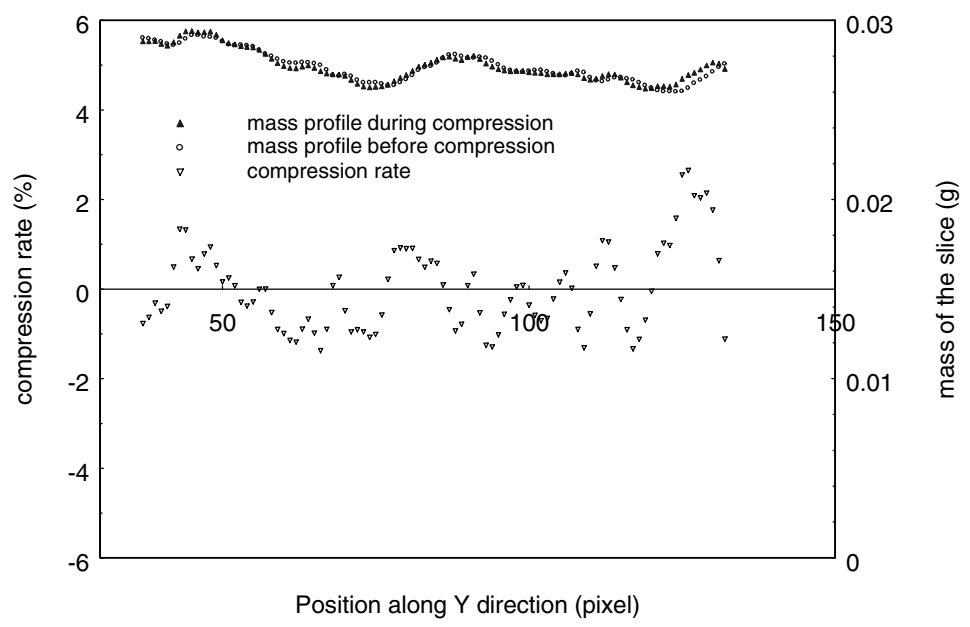

Figure 16. Density (before and during compression) and compression strain profiles (along $Y$ direction).

taken into account. Moreover, the location of binder is certainly a very important parameter that should be taken into account in the understanding the properties of glass wool. Dual-energy experiments could bring some important complementary information. An explorative study is currently being carried out, making use of an x-ray simulation tool developed by the CNDRI laboratory to optimize the dualenergy operating conditions.

We intended to simulate the behaviour of a complete glass wool plate, that is a semi-infinite medium, and this work deals only with $1 \mathrm{D}$ deformation. However, we could expect that tomographic data could also be used in the general case of 3D strain measurements during a mechanical test. A similar principle, using matter conservation, could be considered.

\section{Acknowledgments}

The authors thank Mrs Cantelaube for the supply of the glass wool samples, the calibration samples and the financial support of ISOVER (Saint-Gobain Company). We also thank the CEALETI (Grenoble, France) which allowed the free disposition of the tomographic reconstruction software, and particularly
C Robert-Coutant for the help she provided. The work was carried out in the CNDRI laboratory.

\section{References}

Badel E 1999 Détermination des propriétés élastiques d'éléments individuels du plan ligneux du chêne par des essais de traction sur micro-éprouvettes Ann. For. Sci. 56 467-78

Badel E and Perré P 2001 Using a digital x-ray imaging device to measure the swelling coefficients of a group of wood cells NDT\& E Int. 34 345-53

Baruchel J et al 2000 X-Ray Tomography in Material Science (Paris: Hermes)

Cendre E, Duvauchelle P, Peix G, Buffière J-Y and Babot D 1999 Conception of a high resolution x-ray computed tomography device; application to damage initiation imaging inside materials Proc. 1st World Congr. on Industrial Process Tomography (Buxton, 1999) pp 362-9

Douarche N, Rouby D, Peix G and Jouin J M 2001 Relations between $\mathrm{x}$-ray tomography, specific gravity and mechanical properties in carbon-carbon composites for braking applications Carbon 39 1455-65

Elliott J A, Windle A H, Hobdell J R, Eeckhaut G, Oldman R J, Ludwig W, Boller E, Cloetens P and Baruchel J 2002 In situ deformation of an open-cell flexible polyurethane foam characterised by 3D computed microtomography J. Mater. Sci. 37 1547-55 
Farruggia F and Perré P 2000 Microscopic tensile tests in the transverse plane of earlywood and latewood parts of spruce Wood Sci. Tech. 34 65-82

Feldkamp L A, Davis L C and Kress J w 1984 Practical cone-beam algorithm J. Opt. Soc. 1 612-19

Grangeat P 2001 Fully three-dimensional image reconstruction in radiology and nuclear medicine Encyclopedia of Computer Science and Technology (Suppl. 29) vol 44, ed A Kent and J G Williams (New York: Dekker) ch 10 pp 167-201

Grangeat P 2002 La Tomographie Traité IC2 (Paris: Hermes)

Kak A C and Slaney M 1988 Principles of Computerized Tomographic Imaging (New York: IEEE)

Maire E, Buffiere J-Y, Cloetens P, Ludwig W and Peix G 2002 High resolution $\mathrm{X}$-ray tomography applied in the observation of the micro-structure of materials Prakt. Metallogr. 3 155-68

de Paiva R F, Lynch J, Rosenberg E and Bisiaux M 1998 A beam hardening correction for $\mathrm{x}$-ray microtomography $N D T \& E$ Int 31 17-22
Papadopoulos A N and Traboulay E 2002 Dimensional stability of OSB made from acetylated Fir strands Holz als Roh Werkstoff 60 84-7

Radon J 1917 Über die bestimmung von funktionen durch ihre integralwerte längs gewisser mannigfaltigkeiten Berichte Sächsische Akademie der Wissenschaften, Leipzig, Math-Phys. Kl. 69 262-77

Robert-Coutant C, Moulin V, Sauze R, Rizo P and Casagrande J M 1999 Estimation of the matrix attenuation in heterogeneous radioactive waste drums using dual-energy computed tomography Nucl. Instrum. Methods Phys. Res. A 422 949-56

Tatschl A, Scherer S, Werth P and Kolednik O 2000 Automatic local deformation measurements by means of a digital image analysis system Euromat 2000 Proc. vol 2 pp 899-904

Wang S, Winistorfer P M, Young T M and Helton C 2001 Step-closing pressing of medium density fibreboard. Part 1 : Influence on the vertical density profile Holz als Roh Werkstoff 59 19-26 